Kragujevac Journal of Mathematics

Volume 40(1) (2016), Pages 79-89.

\title{
EXTREMAL ENERGY OF DIGRAPHS WITH TWO LINEAR SUBDIGRAPHS
}

\author{
RASHID FAROOQ ${ }^{1}$, MEHTAB KHAN $^{1}$, AND YASIR MASOOD $^{1}$
}

\begin{abstract}
The eigenvalues of a digraph are the eigenvalues of its adjacency matrix. The sum of the absolute values of the real part of the eigenvalues is called the energy of the digraph. The extremal energy of bicyclic digraphs with vertex-disjoint directed cycles is known. In this paper, we consider a class of bicyclic digraphs with exactly two linear subdigraphs of equal length. We find the minimal and maximal energy among the digraphs in this class.
\end{abstract}

\section{INTRODUCTION}

A graph $G=(\mathcal{V}, \mathcal{E})$ is composed of two finite sets $\mathcal{V}$ and $\mathcal{E}$. The elements of $\mathcal{V}$ are called vertices and the elements of $\mathcal{E}$ are known as edges. A graph is simple if it has neither self loop nor multiple edges. The adjacency matrix of an $n$-vertex simple graph $G$, denoted by $A_{G}$, is a symmetric matrix of order $n \times n$ whose rows and columns are indexed by the vertices of $G$, where $a_{i j}=1$ if there is an edge between vertices $i$ and $j$, otherwise zero. The characteristic polynomial of a graph $G$ is $\Phi_{G}(x)=\operatorname{det}\left(x I-A_{G}\right)$, where $I$ is the identity matrix of order $n \times n$. The eigenvalues of a graph are actually the eigenvalues of its adjacency matrix. The eigenvalues of a graph together with their multiplicities form the spectrum of a graph. The energy of a graph is the sum of absolute values of its eigenvalues. In the literature, we can find several results on the bounds for energy of graphs. The reader is referred to $[3-5,11]$ for a comprehensive study on the bounds for the energy of bipartite graphs, trees and benzenoids. Hou [6] addressed the problem of finding the uncyclic graphs with minimal energy. Hou et al. [7] considered the problem of finding unicyclic graphs with maximal energy. They

Key words and phrases. Energy of a digraph, bicyclic digraphs, linear subdigraphs.

2010 Mathematics Subject Classification. Primary: 05C35, 05C50.

Received: July 16, 2015.

Accepted: February 17, 2016. 
find unicyclic graphs with maximum energy among all unicyclic graphs with fixed number of vertices and with fixed length of cycles.

Pena and Rada [9] extended the concept of energy to digraphs. The authors find the unicyclic digraphs which have minimal and maximal energy among all unicyclic digraphs with fixed number of vertices. Adiga et al. [1] introduced the notion of skew energy of digraphs, which is the sum of absolute values of the eigenvalues of the skew-adjacency matrix of the digraph. The authors derive an upper bound for the skew energy of digraph and computed skew energies of directed cycles. Pirzada and Bhat [10] extended the concept of energy of digraphs to the energy of signed digraphs. The authors extended the notion of NEPS to signed digraphs and constructed infinite families of non cospectral equienergetic signed digraphs. In Khan et al. [8], the authors studied extremal energy of bicyclic digraphs with vertex-disjoint directed cycles. In the present paper, we extend the same problem to the class of bicyclic digraphs with exactly two linear subdigraphs of equal length and find minimal and maximal energy for these digraphs.

\section{Preliminaries}

First, we give some definitions and terminologies. A directed graph (or digraph) is defined to be a pair $D=(\mathcal{V}, \mathcal{A})$, the elements of $\mathcal{V}$ are called vertices and the elements of $\mathcal{A}$ are called arcs. If there is an arc from a vertex $u$ to a vertex $v$, we denote it by $u v$. A directed cycle $C_{n}$ of length $n(n \geq 2)$ is a digraph with vertex set $\left\{v_{i} \mid i=1, \ldots, n\right\}$ of $n$ elements and arc set $\left\{v_{i} v_{i+1} \mid i=1, \ldots, n-1\right\} \cup\left\{v_{n} v_{1}\right\}$ of $n$ elements. A digraph is connected if its underlying graph is connected. A unicyclic digraph is a connected digraph which contains a unique directed cycle. A bicyclic digraph is a connected digraph which contains exactly two directed cycles.

The adjacency matrix $A_{G}=\left[a_{i j}\right]_{n \times n}$ of an $n$-vertex digraph $D=(\mathcal{V}, \mathcal{A})$ is defined as

$$
a_{i j}=\left\{\begin{array}{ll}
1, & \text { if } v_{i} v_{j} \in \mathcal{A}, \\
0, & \text { otherwise }
\end{array} \quad \text { for all } v_{i}, v_{j} \in \mathcal{V} .\right.
$$

For digraphs, the eigenvalues may be complex numbers since the adjacency matrix of a digraph is not necessarily symmetric. The energy of a digraph is defined as the sum of the absolute values of the real parts of its eigenvalues. That is, if $z_{1}, z_{2}, \ldots, z_{n}$ are eigenvalues of an $n$-vertex digraph $D$, then energy of $D$ is defined by

$$
E(D)=\sum_{i=1}^{n}\left|\operatorname{Re}\left(z_{i}\right)\right|,
$$

where $\operatorname{Re}\left(z_{i}\right)$ is the real part of $z_{i}$.

Theorem 2.1 (Coefficients theorem for digraphs [2]). Let D be an n-vertex digraph with characteristic polynomial given by

$$
\Phi_{D}(x)=x^{n}+\sum_{k=1}^{n} b_{k} x^{n-k} .
$$




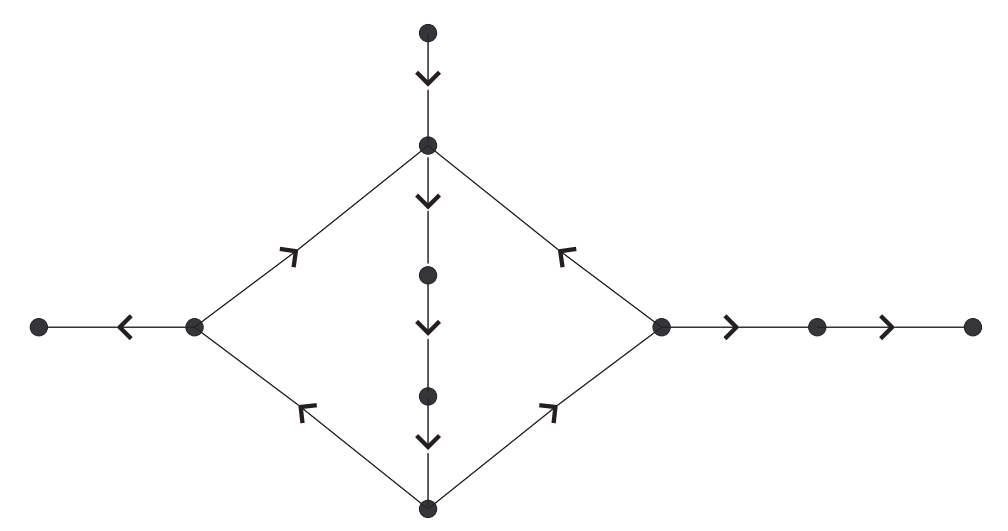

Figure 1. A digraph $D \in \mathcal{D}_{10}$.

Then $b_{k}=\sum_{L \in \mathcal{L}_{k}}(-1)^{c(L)}$ for each $k=1,2, \ldots, n$, where $\mathcal{L}_{k}$ is the set of all linear subdigraphs $L$ of $D$ with exactly $k$ vertices; $c(L)$ denotes the number of components of $L$.

For $n \geq 3$, we define a set $\mathcal{D}_{n}$ which consists of $n$-vertex digraphs with exactly two linear subdigraphs of equal length. Let $D \in \mathcal{D}_{n}$ be a digraph such that the length of both linear subdigraphs is $m, 2 \leq m<n-1$. From Theorem 2.1, the characteristic polynomial of $D$ is given by

$$
\Phi_{D}(x)=x^{n-m}\left(x^{m}-2\right) .
$$

The eigenvalues of $D$ are 0 and $\sqrt[m]{2} \exp \left(\frac{2 k \pi i}{m}\right)$, where $k=0,1, \ldots, m-1$ and the multiplicity of eigenvalue 0 is $n-m$. Thus the energy of $D$ is given by

$$
E(D)=\sqrt[m]{2} \sum_{k=0}^{m-1}\left|\cos \frac{2 k \pi}{m}\right|
$$

Example 2.1. Consider the digraph $D \in \mathcal{D}_{10}$ shown in Figure 1. Using Theorem 2.1, the characteristic polynomial of $D$ is given by

$$
\Phi_{D}(x)=x^{5}\left(x^{5}-2\right) .
$$

The eigenvalues of $D$ are 0 and $\sqrt[5]{2} \exp \left(\frac{2 k \pi i}{5}\right), k=0,1, \ldots, 4$, and the multiplicity of 0 is 5 . Thus, the energy of $D$ is

$$
E(D)=\sqrt[5]{2} \sum_{k=0}^{4}\left|\cos \frac{2 k \pi}{5}\right|
$$


From (2.1), we compute the energy formulae of $D$ as follows:

$$
E(D)= \begin{cases}2 \sqrt[m]{2} \cot \frac{\pi}{m}, & \text { if } m \equiv 0(\bmod 4), \\ 2 \sqrt[m]{2} \csc \frac{\pi}{m}, & \text { if } m \equiv 2(\bmod 4), \\ \sqrt[m]{2} \csc \frac{\pi}{2 m}, & \text { if } m \equiv 1(\bmod 2) .\end{cases}
$$

Lemma 2.1 (Khan et al. [8]). Let $x, a, b$ be real numbers such that $x \geq a>0$ and $b>0$. Then we have

$$
\frac{\pi x}{b x^{2}-\pi^{2}} \leq \frac{\pi a}{b a^{2}-\pi^{2}} .
$$

For any real number $x$ with $0<x<\frac{\pi}{2}$, the following inequalities hold:

$$
\begin{gathered}
\sin x \leq x, \quad \sin x \geq x-\frac{x^{3}}{3 !}, \\
\cot x \leq \frac{1}{x}, \quad \cot x \geq \frac{1}{x}-\frac{x}{2}, \quad \text { if } x \neq 0 .
\end{gathered}
$$

\section{Extremal ENERGy}

Pena and Rada [9] considered the problem of finding extremal energy among all unicyclic digraphs with fixed number of vertices. Khan et al. [8] considered the same problem for finding extremal energy for bicyclic digraphs with vertex-disjoint directed cycles. In this section, we find the minimal and maximal energy among the digraphs in $\mathcal{D}_{n}$. The following lemma gives us the lower bound for the energy of a digraph $D \in \mathcal{D}_{n}$.

Lemma 3.1. Let $D \in \mathcal{D}_{n}$ such that the length of each linear subdigraph is $m$, where $2 \leq m \leq n-1$. Then

$$
E(D) \geq \begin{cases}\sqrt[m]{2}\left(\frac{2 m}{\pi}-\frac{\pi}{m}\right), & \text { if } m \equiv 0(\bmod 4) \\ \sqrt[m]{2}\left(\frac{2 m}{\pi}\right), & \text { otherwise. }\end{cases}
$$

Proof. Let $m \equiv 0(\bmod 4)$. Then by $(2.2)$ and $(2.4)$, we get

$$
\begin{aligned}
E(D) & =2 \sqrt[m]{2}\left(\cot \frac{\pi}{m}\right) \\
& \geq 2 \sqrt[m]{2}\left(\frac{m}{\pi}-\frac{\pi}{2 m}\right) \\
& =\sqrt[m]{2}\left(\frac{2 m}{\pi}-\frac{\pi}{m}\right) .
\end{aligned}
$$


Next, if $m \equiv 2(\bmod 4)$ then $(2.2)$ and $(2.3)$ yield

$$
\begin{aligned}
E(D) & =2 \sqrt[m]{2}\left(\csc \frac{\pi}{m}\right) \\
& =2 \sqrt[m]{2}\left(\frac{1}{\sin \frac{\pi}{m}}\right) \\
& \geq \sqrt[m]{2}\left(\frac{2 m}{\pi}\right)
\end{aligned}
$$

Analogously, if $m \equiv 1(\bmod 2)$ then we can show that

$$
E(D) \geq \sqrt[m]{2}\left(\frac{2 m}{\pi}\right)
$$

The proof is complete.

The following lemma gives the upper bound for the energy of a digraph $D \in \mathcal{D}_{n}$.

Lemma 3.2. Let $D \in \mathcal{D}_{n}$ such that the length of each linear subdigraph is $m$ where $2 \leq m \leq n-1$. Then

$$
E(D) \leq \begin{cases}\sqrt[m]{2}\left(\frac{2 m}{\pi}\right), & \text { if } m \equiv 0(\bmod 4) \\ \sqrt[m]{2}\left(\frac{2 m}{\pi}+\frac{2 m \pi}{6 m^{2}-\pi^{2}}\right), & \text { if } m \equiv 2(\bmod 4) \\ \sqrt[m]{2}\left(\frac{2 m}{\pi}+\frac{2 m \pi}{24 m^{2}-\pi^{2}}\right), & \text { if } m \equiv 1(\bmod 2)\end{cases}
$$

Proof. First, let $m \equiv 0(\bmod 4)$. By $(2.2)$ and $(2.4)$, we get

$$
\begin{aligned}
E(D) & =2 \sqrt[m]{2} \cot \frac{\pi}{m} \\
& \leq 2 \sqrt[m]{2}\left(\frac{m}{\pi}\right) \\
& =\sqrt[m]{2}\left(\frac{2 m}{\pi}\right)
\end{aligned}
$$


Next, we take $m \equiv 2(\bmod 4)$. By $(2.2)$ and $(2.3)$, we get

$$
\begin{aligned}
E(D) & =2 \sqrt[m]{2} \csc \frac{\pi}{m} \\
& \leq 2 \sqrt[m]{2}\left(\frac{1}{\frac{\pi}{m}\left(1-\frac{\pi^{2}}{6 m^{2}}\right)}\right) \\
& =2 \sqrt[m]{2}\left(\frac{m}{\pi}\left(\frac{6 m^{2}}{6 m^{2}-\pi^{2}}\right)\right) \\
& =2 \sqrt[m]{2}\left(\frac{m}{\pi}\left(1+\frac{\pi^{2}}{6 m^{2}-\pi^{2}}\right)\right) \\
& =\sqrt[m]{2}\left(\frac{2 m}{\pi}+\frac{2 m \pi}{6 m^{2}-\pi^{2}}\right)
\end{aligned}
$$

Finally, if $m \equiv 1(\bmod 2)$ then one can analogously show that

$$
E(D) \leq \sqrt[m]{2}\left(\frac{2 m}{\pi}+\frac{2 m \pi}{24 m^{2}-\pi^{2}}\right)
$$

This completes the proof.

Next lemma shows that the energy of digraphs in $\mathcal{D}_{n}$ increases monotonically with the increase in length of their linear subdigraphs.

Lemma 3.3. Let $n \geq 6$ and $5 \leq m \leq n-1$. Take $D \in \mathcal{D}_{n}$ with length of each linear subdigraph $m+1$ and $D^{\prime} \in \mathcal{D}_{n}$ with length of each linear subdigraph $m$. Then

$$
E(D) \geq E\left(D^{\prime}\right)
$$

Proof. Let $m \equiv 0(\bmod 4)$. In this case, $m \geq 8$ and $m+1 \equiv 1(\bmod 2)$. Lemma 3.1 implies

$$
E(D) \geq \sqrt[m+1]{2}\left(\frac{2 m}{\pi}+\frac{2}{\pi}\right)
$$

On the other hand, Lemma 3.2 gives

$$
E\left(D^{\prime}\right) \leq \sqrt[m]{2}\left(\frac{2 m}{\pi}\right)
$$

One can easily see that the following inequality holds:

$$
\sqrt[m+1]{2}\left(\frac{2 m}{\pi}+\frac{2}{\pi}\right) \geq \sqrt[m]{2}\left(\frac{2 m}{\pi}\right) .
$$

Combining (3.1)-(3.3), we get

$$
E(D) \geq E\left(D^{\prime}\right)
$$


Next, let $m \equiv 2(\bmod 4)$. In this case $m \geq 6$ and $m+1 \equiv 1(\bmod 2)$. Lemma 3.1 implies that

$$
E(D) \geq \sqrt[m+1]{2}\left(\frac{2 m}{\pi}+\frac{2}{\pi}\right)
$$

On the other hand, Lemma 3.2 gives

$$
E\left(D^{\prime}\right) \leq \sqrt[m]{2}\left(\frac{2 m}{\pi}+\frac{2 m \pi}{6 m^{2}-\pi^{2}}\right)
$$

Furthermore, Lemma 2.1 implies that

$$
\sqrt[m]{2}\left(\frac{2 m}{\pi}+\frac{2 m \pi}{6 m^{2}-\pi^{2}}\right) \leq \sqrt[m]{2}\left(\frac{2 m}{\pi}+\frac{12 \pi}{6^{3}-\pi^{2}}\right)
$$

One can easily see that the following inequality holds:

$$
\sqrt[m+1]{2}\left(\frac{2 m}{\pi}+\frac{2}{\pi}\right) \geq \sqrt[m]{2}\left(\frac{2 m}{\pi}+\frac{12 \pi}{6^{3}-\pi^{2}}\right)
$$

Combining (3.4)-(3.7), we get

$$
E(D) \geq E\left(D^{\prime}\right)
$$

Finally, let $m \equiv 1(\bmod 2)$. In this case $m \geq 7, m+1 \equiv 2(\bmod 4)$ or $m+1 \equiv$ $0(\bmod 4)$.

If $m+1 \equiv 2(\bmod 4)$ then Lemma 3.1 implies

$$
E(D) \geq \sqrt[m+1]{2}\left(\frac{2 m}{\pi}+\frac{2}{\pi}\right)
$$

On the other hand, Lemma 3.2 gives

$$
E\left(D^{\prime}\right) \leq \sqrt[m]{2}\left(\frac{2 m}{\pi}+\frac{2 m \pi}{24 m^{2}-\pi^{2}}\right)
$$

Lemma 2.1 implies that

$$
\sqrt[m]{2}\left(\frac{2 m}{\pi}+\frac{2 m \pi}{24 m^{2}-\pi^{2}}\right) \leq \sqrt[m]{2}\left(\frac{2 m}{\pi}+\frac{14 \pi}{24.7^{2}-\pi^{2}}\right) .
$$

The following inequality is easily seen:

$$
\sqrt[m+1]{2}\left(\frac{2 m}{\pi}+\frac{2}{\pi}\right) \geq \sqrt[m]{2}\left(\frac{2 m}{\pi}+\frac{14 \pi}{24.7^{2}-\pi^{2}}\right)
$$

Combining (3.8)-(3.11), we get

$$
E(D) \geq E\left(D^{\prime}\right) \text {. }
$$

If $m+1 \equiv 0(\bmod 4)$, one can analogously show that

$$
E(D) \geq E\left(D^{\prime}\right) \text {. }
$$

This completes the proof.

The following corollary is an easy consequence of Lemma 3.3. 
Corollary 3.1. Let $n \geq 6$ and $2 \leq m_{2} \leq m_{1} \leq n-1$. Take $D \in \mathcal{D}_{n}$ with length of each linear subdigraph $m_{1}$ and $D^{\prime} \in \mathcal{D}_{n}$ with length of each linear subdigraph $m_{2}$. Then

$$
E(D) \geq E\left(D^{\prime}\right)
$$

Using Corollary 3.1, we finally prove the main theorem.

Theorem 3.1. Let $n \geq 6$ and $D \in \mathcal{D}_{n}$ be a digraph with length of each linear subdigraph $m$, where $2 \leq m \leq n-1$. Then $D$ has minimal energy when $m=4$ and maximal energy when $m=n-1$ among all digraphs of $\mathcal{D}_{n}$.

Proof. Let $D \in \mathcal{D}_{n}$ be a digraph with linear subdigraphs of equal length $m$ where $2 \leq m \leq n-1$. Then it can easily be seen from (2.2) that

$$
E(D)= \begin{cases}2 \sqrt[2]{2}, & \text { if } m=2 \\ 2 \sqrt[3]{2}, & \text { if } m=3 \\ 2 \sqrt[4]{2}, & \text { if } m=4\end{cases}
$$

However, if $m \geq 5$, then from Corollary 3.1, we get

$$
2 \sqrt[2]{2} \leq E(D) \leq E(H)
$$

where $H$ is a digraph with linear subdigraphs of equal length $r, r \geq m$. It is clear from (3.12) and (3.13) that $E(D)$ is minimal when $m=4$. Furthermore, $\mathcal{D}_{n}$ contains a digraph with maximum length of each linear subdigraph $n-1$. Therefore, from (3.13) it is evident that $E(D)$ is maximal when $m=n-1$.

\section{Comparison of Extremal Energies}

Let $\mathcal{B}_{n}$ be a set consisting of $n$-vertex bicyclic digraphs such that directed cycles in a digraph are vertex-disjoint, $n \geq 4$. In Khan et al. [8], the authors study the problem of finding minimal and maximal energy among the digraphs in $\mathcal{B}_{n}$. For a digraph $G \in \mathcal{B}_{n}$ with vertex-disjoint directed cycles $C_{n-2}$ and $C_{2}$, the following lemma gives lower bound on the energy of $G$.

Lemma 4.1 (Khan et al. [8]). Let $n \geq 4$ and $G \in \mathcal{B}_{n}$ with vertex-disjoint directed cycles $C_{n-2}$ and $C_{2}$. Then we have the following inequalities:

$$
E(G)=E\left(C_{n-2}\right)+E\left(C_{2}\right) \geq \begin{cases}\frac{2 n}{\pi}-\frac{4}{\pi}-\frac{\pi^{2}}{3(n-2)^{2}}+2, & \text { if } n \equiv 0(\bmod 4), \\ \frac{2 n}{\pi}-\frac{4}{\pi}-\frac{\pi}{n-2}+2, & \text { if } n \equiv 2(\bmod 4), \\ \frac{2 n}{\pi}-\frac{4}{\pi}-\frac{\pi^{2}}{12(n-2)^{2}}+2, & \text { if } n \equiv 1(\bmod 2) .\end{cases}
$$

Theorem 4.1 (Khan et al. [8]). Let $G \in \mathcal{D}_{n}$ with directed cycles $C_{r_{1}}$ and $C_{r_{2}}$, where $2 \leq r_{1}, r_{2} \leq n-2$. Then $G$ has minimal energy if $2 \leq r_{1}, r_{2} \leq 4$ and maximal energy if $r_{1}=n-2$ and $r_{2}=2$ or the vice versa. 


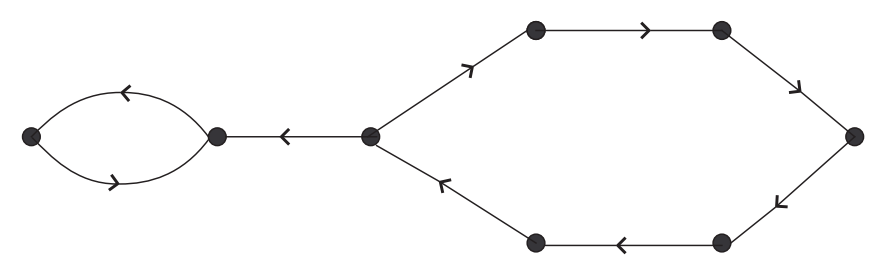

FigURE 2. A digraph with maximal energy in $\mathcal{B}_{8}$.

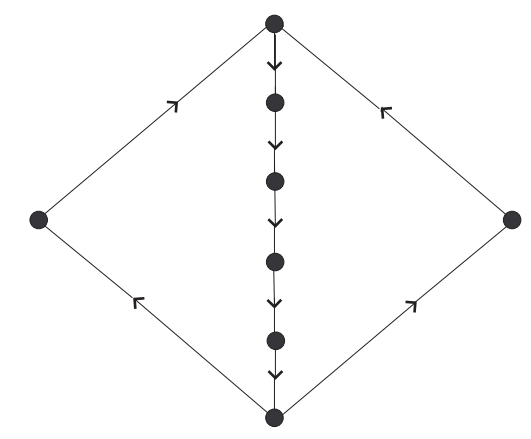

Figure 3. A digraph with maximal energy in $D_{8}$.

In the following theorem, we compare the maximal energy among the digraphs in $\mathcal{B}_{n}$ and $\mathcal{D}_{n}$. A digraph with maximal energy in $\mathcal{B}_{8}$ is shown in Figure 2 and a digraph with maximal energy in $D_{8}$ is shown in Figure 3.

Theorem 4.2. Let $n \geq 6$. Then the maximal energy among the digraphs in $\mathcal{B}_{n}$ is always greater than the maximal energy among the digraphs in $\mathcal{D}_{n}$.

Proof. Let $G \in \mathcal{B}_{n}$ with vertex-disjoint directed cycles $C_{n-2}$ and $C_{2}$. Also take $D \in \mathcal{D}_{n}$ with linear subdigraphs of equal length $n-1$.

Let $n \equiv 0(\bmod 4)$. In this case we have $n-2 \geq 6$ and from Lemma 4.1 we have

$$
\begin{aligned}
E(G)=E\left(C_{n-2}\right)+E\left(C_{2}\right) & \geq \frac{2 n}{\pi}-\frac{4}{\pi}-\frac{\pi^{2}}{3(n-2)^{2}}+2 \\
& \geq \frac{2 n}{\pi}-\frac{4}{\pi}-\frac{\pi^{2}}{108}+2 .
\end{aligned}
$$

On the other hand, from Lemma 3.2 we have

$$
E(D) \leq \sqrt[n-1]{2}\left(\frac{2 n}{\pi}-\frac{2}{\pi}+\frac{2(n-1) \pi}{24(n-1)^{2}-\pi^{2}}\right) .
$$

By using Lemma 2.1 we have

$$
\sqrt[n-1]{2}\left(\frac{2 n}{\pi}-\frac{2}{\pi}+\frac{2(n-1) \pi}{24(n-1)^{2}-\pi^{2}}\right) \leq \sqrt[n-1]{2}\left(\frac{2 n}{\pi}-\frac{2}{\pi}+\frac{14 \pi}{24(7)^{2}-\pi^{2}}\right)
$$


One can easily see that the following inequality holds:

$$
\frac{2 n}{\pi}-\frac{4}{\pi}-\frac{\pi^{2}}{108}+2 \geq \sqrt[n-1]{2}\left(\frac{2 n}{\pi}-\frac{2}{\pi}+\frac{14 \pi}{24(7)^{2}-\pi^{2}}\right) .
$$

Inequalities (4.1)-(4.4) imply

$$
E(G) \geq E(D)
$$

Next, let $n \equiv 2(\bmod 4)$. In this case we have $n-2 \geq 4$ and from Lemma 4.1 we have

$$
\begin{aligned}
E(G)=E\left(C_{n-2}\right)+E\left(C_{2}\right) & \geq \frac{2 n}{\pi}-\frac{4}{\pi}-\frac{\pi}{n-2}+2 \\
& \geq \frac{2 n}{\pi}-\frac{4}{\pi}-\frac{\pi}{4}+2 .
\end{aligned}
$$

On the other hand, we have from Lemma 3.2 that

$$
E(D) \leq \sqrt[n-1]{2}\left(\frac{2 n}{\pi}-\frac{2}{\pi}+\frac{2(n-1) \pi}{24(n-1)^{2}-\pi^{2}}\right) .
$$

By using Lemma 2.1 we have

$$
\sqrt[n-1]{2}\left(\frac{2 n}{\pi}-\frac{2}{\pi}+\frac{2(n-1) \pi}{24(n-1)^{2}-\pi^{2}}\right) \leq \sqrt[n-1]{2}\left(\frac{2 n}{\pi}-\frac{2}{\pi}+\frac{14 \pi}{24(7)^{2}-\pi^{2}}\right) .
$$

The following inequality holds true:

$$
\frac{2 n}{\pi}-\frac{4}{\pi}-\frac{\pi}{4}+2 \geq \sqrt[n-1]{2}\left(\frac{2 n}{\pi}-\frac{2}{\pi}+\frac{14 \pi}{24(7)^{2}-\pi^{2}}\right) .
$$

Inequalities (4.5) and (4.6) imply

$$
E(G) \geq E(D)
$$

Finally, let $n \equiv 1(\bmod 2)$. In this case we have $n-2 \geq 5$ and from Lemma 4.1 we have

$$
\begin{aligned}
E(G)=E\left(C_{n-2}\right)+E\left(C_{2}\right) & \geq \frac{2 n}{\pi}-\frac{4}{\pi}-\frac{\pi^{2}}{12(n-2)^{2}}+2 \\
& \geq \frac{2 n}{\pi}-\frac{4}{\pi}-\frac{\pi^{2}}{12(5)^{2}}+2 .
\end{aligned}
$$

On the other hand, we have from Lemma 3.2 that

$$
E(D) \leq \sqrt[n-1]{2}\left(\frac{2 n}{\pi}-\frac{4}{\pi}\right)
$$

The following inequality is easily seen:

$$
\frac{2 n}{\pi}-\frac{4}{\pi}-\frac{\pi^{2}}{12(5)^{2}}+2 \geq \sqrt[n-1]{2}\left(\frac{2 n}{\pi}-\frac{4}{\pi}\right)
$$

From inequalities (4.7) and (4.8), we get

$$
E(G) \geq E(D) .
$$

Thus, from Theorem 3.1 and Theorem 4.2, the assertion is true. 


\section{Conclusion}

In this paper, we introduced a new class $\mathcal{D}_{n}$ of bicyclic digraphs. Each $D \in \mathcal{D}_{n}$ has exactly two linear subdigraphs of equal length. For $n \geq 6$, we showed that $D \in \mathcal{D}_{n}$ has maximal energy among the digraphs of $\mathcal{D}_{n}$ if the length of each linear subdigraph is $n-1$. Furthermore, $D$ has minimal energy if length of each subdigraph is 4 . It will be interesting to consider a more general class of bicyclic digraphs where the length of each linear subdigraph is not necessarily equal. We leave this as an open problem.

\section{REFERENCES}

[1] C. Adiga, R. Balakrishnan and W. So, The skew energy of a digraph, Linear Algebra Appl. 432 (2010), 1825-1835.

[2] D. M. Cvetkovic, M. Doob and H. Sachs, Spectra of Graphs, Academic Press, New York, 1980.

[3] I. Gutman, Total $\pi$-electron energy of benzenoid hydrocarbon, Topic Curr. Chem. 162 (1992), 29-63.

[4] I. Gutman, Acyclic conjugated molecules, trees and their energies, J. Math. Chem. 2 (1987), $123-143$.

[5] I. Gutman, Acyclic systems with extremal Hückel $\pi$-electron energy, Theor. Chim. Acta 45 (1977), 79-87.

[6] Y. Hou, Unicyclic graphs with minimal energy, J. Math. Chem. 29 (3) (2001), 163-168.

[7] Y. Hou, I. Gutman and C. W. Woo, Unicyclic graphs with maximal energy, Linear Algebra Appl. 356 (2002), 27-36.

[8] M. Khan, R. Farooq and A. A. Siddiqui, On the extremal energy of bicyclic digraphs, J. Math. Inequal. 9 (3) (2015), 799-810.

[9] I. Pena and J. Rada, Energy of digraphs, Linear Multilinear Algebra 56 (5) (2008), 565-579.

[10] S. Pirzada and M. A. Bhat, Energy of signed digraphs, Discrete Appl. Math. 169 (2014), $195-205$.

[11] L. Turker, An upper bound for total $\pi$-electron energy of alternant hydrocarbons, MATCH Commun. Math. Comput. Chem. 6 (1984), 83-94.

${ }^{1}$ School of Natural Sciences,

National University of Sciences and Technology,

H-12 Islamabad, Pakistan

E-mail address: farook.ra@gmail.com (R. Farooq), mehtabkhan85@gmail.com (M. Khan), yasir_masood890yahoo.com (Y. Masood) 